\title{
The Impact of the COVID-19 Pandemic on Long-Term Care in Canada
}

Raquel Betini, Sandra Milicic and Christina Lawand

\begin{abstract}
The COVID-19 pandemic has disproportionately affected Canada's long-term care (LTC) sector, with residents of LTC and retirement homes accounting for $67 \%$ of all COVID-19-related deaths as of February 15, 2021. This study investigated the impact of the COVID-19 pandemic on LTC residents across Canada during the first six months of the pandemic, including how care changed for residents, using data from the Canadian Institute for Health Information's LTC and acute care databases. The results suggest that LTC residents received less medical care, with fewer physician visits and hospital transfers compared with the same period in 2019. They also had less contact with family/friends compared with the same period in 2019, which was associated with higher levels of depression. In provinces where it could be measured, the number of LTC resident deaths from all causes was higher than pre-pandemic years during the peak of the first wave, even in jurisdictions with few COVID-19related deaths in LTC.
\end{abstract}

\section{Introduction}

The COVID-19 pandemic exposed long-standing issues in Canada's long-term care (LTC) sector, such as underpaid staff, overcrowding, insufficient personal protective equipment and other factors related to resident care. These issues, combined with the infectious nature of the COVID-19 virus, caused the perfect storm for the quick spread of infection in LTC. The impact of the COVID-19 pandemic on LTC residents and staff was devastating, with high death rates. Strict lockdown measures in the first wave also led to changes in how residents received medical care and interacted with their family (CIHI 2020, 2021).

\begin{abstract}
Approach
Our results use a combination of data from the Canadian Institute for Health Information's (CIHI's) Continuing Care Reporting System, Discharge Abstract Database and National Ambulatory Care Reporting System. We define the first wave of the COVID-19 pandemic as the period from March 1 to August 31, 2020, with a peak between March 1 and June 30, and compare care and outcome patterns to the same period in 2019. A five-year average (2015-2019) period was used as a pre-pandemic comparison time frame for excess death calculations.
\end{abstract}

\section{Key Findings}

LTC residents received less medical care and had less contact with family and friends early in the pandemic In Canada, LTC resident transfers to hospitals for the treatment of chronic conditions and infections dropped by $27 \%$ between March and August 2020 compared to 2019 (Figure 1). The largest decrease in hospital transfers was for chronic medical conditions that may require a doctor's order to seek hospital care, such as chronic obstructive pulmonary disease or heart failure (Table 1). Although the reasons are not found in the data, a similar drop has been previously reported for hospitalizations and emergency department visits related to non-urgent care and for more serious health concerns, indicating changes in access to care related to the pandemic (Dudevich and Frood 2021).

Changes to the care provided to residents living in LTC facilities also occurred during the beginning of the pandemic, although the reason behind them is less clear. Compared with the same period in 2019, the proportion of LTC residents who received a doctor's visit declined by $16 \%$, with the largest drops observed in April (24\%) and May (20\%). 
FIGURE 1.

Number of LTC residents transferred to hospital during the first wave* compared with the same period in 2019 in Canada ${ }^{5}$

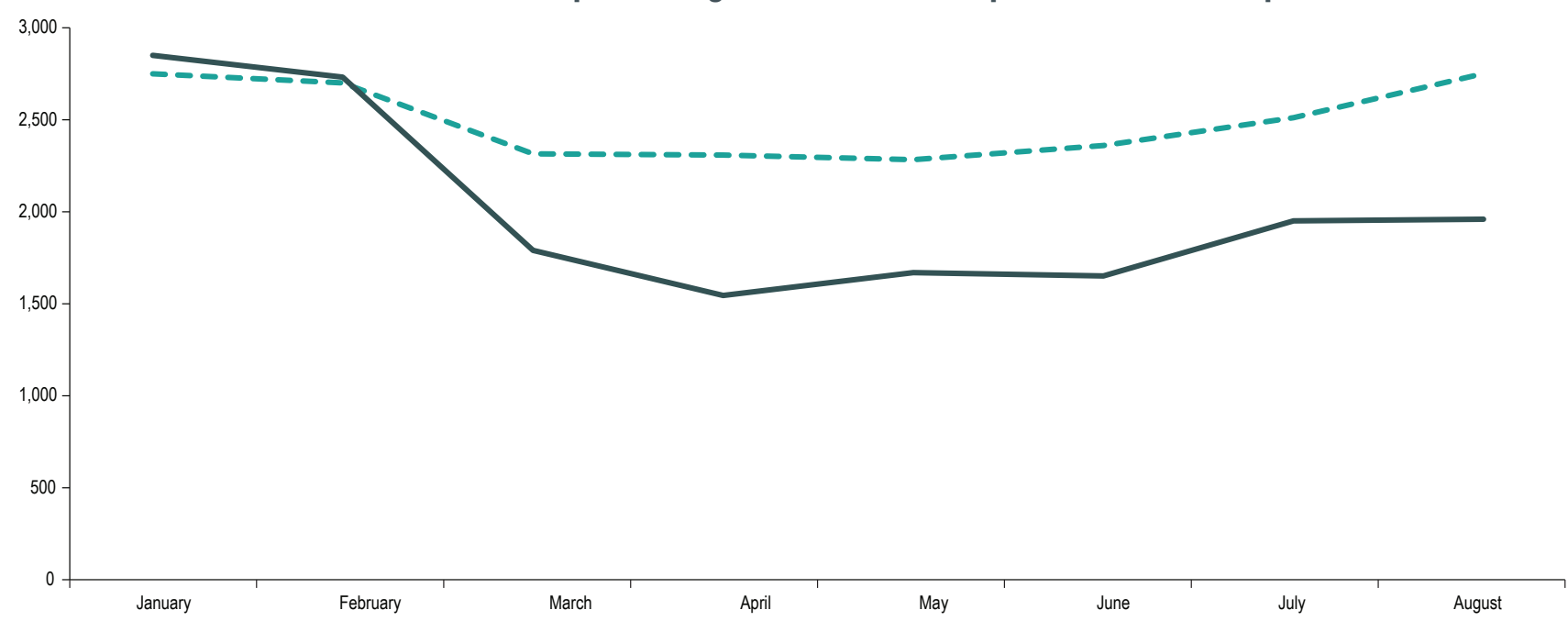

$2020 \quad-\quad 2019$

* The first wave: March 1 to August 31, 2020

$\S$ Quebec data are not included.

Data for LTC transfers are not available for all jurisdictions prior to 2019 for comparison.

Source: Discharge Abstract Database 2018-2019 and 2020-2021, CIHI.

In Canada, as well as in other countries around the world, LTC homes were closed to family and friends to avoid the transmission of COVID-19 (Chu et al. 2021; Stall et al. 2020). Unfortunately, these policies were often related to resident social isolation and poor well-being (Stall et al. 2020). Our data show that the proportion of residents who had no contact with friends or family almost tripled in the first wave, compared with that in 2019 ( $4 \%$ vs $11 \%$ ), and that these residents were more likely to be assessed with depression. Limited visitation likely also had an impact on staff as many visitors were caregivers who provided help with personal care activities such as bathing, eating and dressing (Kemp 2020).

\section{The number of LTC resident deaths was higher than usual during the first wave of the pandemic}

To better understand the impact of the pandemic on resident deaths, this study examined excess deaths. Measuring excess deaths can help to determine whether the deaths observed were expected (i.e., the resident would likely have died from something else), whether COVID-19 caused an unusual increase in deaths or whether a deterioration in the physical and mental health of residents during the pandemic hastened their death, even if they did not have COVID-19. We found that there was a $19 \%$ increase in excess deaths during the peak of the first wave $-2,273$ more deaths than the average in the five years prior to the pandemic (Figure 2). The largest increase in deaths from all causes occurred in April 2020. The COVID-19 pandemic and the lockdown measures had a profound impact on the residents of LTC homes, particularly in the excess mortality observed even in jurisdictions with few COVID-19 deaths and outbreaks.
TABLE 1.

Top 10 reasons for LTC resident transfer to hospital, peak of first wave* compared with 2019 in Canada ${ }^{5}$

\begin{tabular}{|c|c|c|c|}
\hline $\begin{array}{l}\text { Main diagnosis" for } \\
\text { hospital admission }\end{array}$ & 2019 & 2020 & $\begin{array}{l}\text { Percentage } \\
\text { change }\end{array}$ \\
\hline Hip fracture & 667 & 670 & $<1$ \\
\hline COVID-19 infection & $\mathrm{n} / \mathrm{a}$ & 595 & $\mathrm{n} / \mathrm{a}$ \\
\hline Palliative care & 532 & 442 & -17 \\
\hline Urinary tract infection & 542 & 349 & -36 \\
\hline $\begin{array}{l}\text { Sepsis (bloodstream } \\
\text { infection) }\end{array}$ & 464 & 305 & -34 \\
\hline $\begin{array}{l}\text { Pneumonitis due to food } \\
\text { and vomit }\end{array}$ & 520 & 288 & -45 \\
\hline $\begin{array}{l}\text { Pneumonia, organism } \\
\text { unspecified }\end{array}$ & 557 & 276 & -50 \\
\hline Heart failure & 517 & 254 & -51 \\
\hline $\begin{array}{l}\text { Chronic obstructive } \\
\text { pulmonary disease }\end{array}$ & 514 & 216 & -58 \\
\hline Delirium & 201 & 129 & -36 \\
\hline
\end{tabular}

* Peak of the first wave (March 1 to June 30, 2020), compared with the same period in 2019

${ }^{\S}$ Quebec data are not included.

"The acute care diagnosis was used to represent the main diagnosis. $\mathrm{n} / \mathrm{a}=$ not applicable.

Source: Discharge Abstract Database 2019-2020, CIHI 
FIGURE 2.

Excess deaths* of LTC residents during the peak of the first wave ${ }^{5}$ in selected provinces"

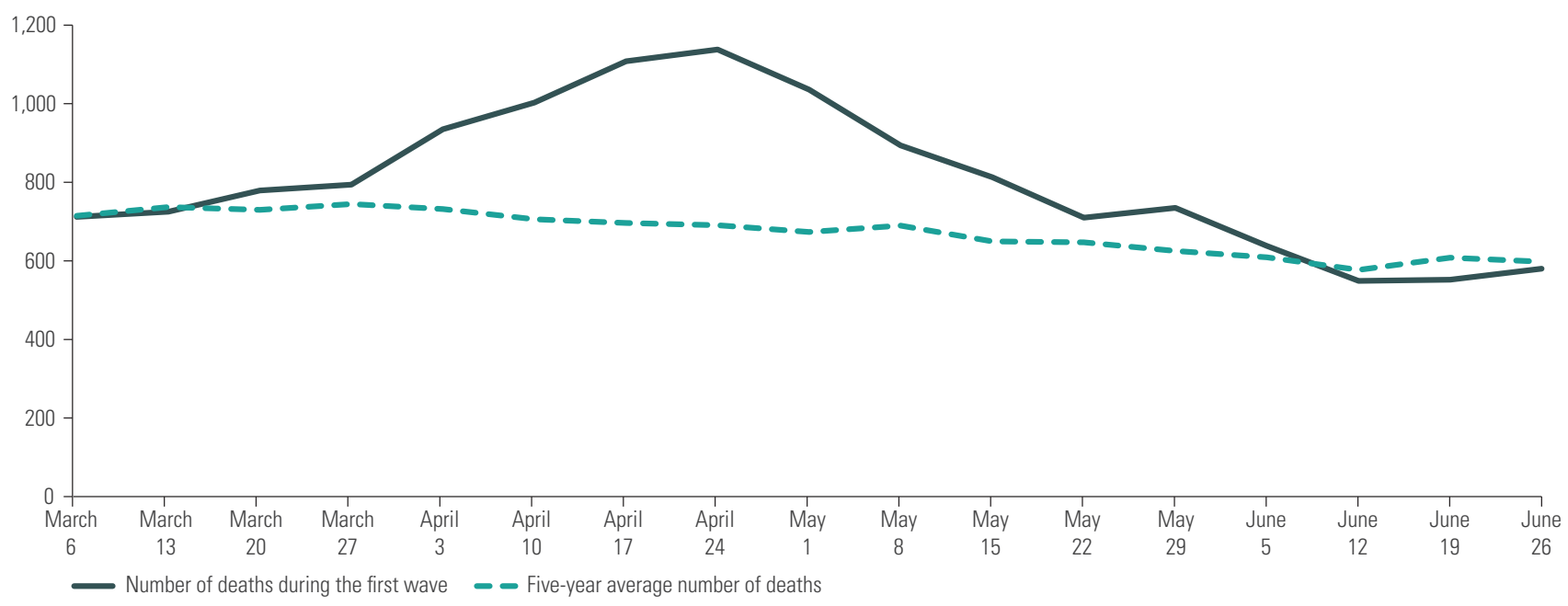

* Excess LTC resident deaths is determined by comparing the number of deaths that occurred between March 1 and June 30, 2020, with the average number of deaths that occurred during the same period in the previous five years (2015 to 2019). Deaths include death from all causes and represent the LTC residents who died in the LTC home or in a hospital.

§ Peak of first wave: March 1 to June 30, 2020.

"Only the provinces that report to the Continuing Care Reporting System are included in this analysis (Newfoundland and Labrador, Ontario, Manitoba, Alberta and British Columbia).

Sources: Continuing Care Reporting System, Discharge Abstract Database and National Ambulatory Care Reporting System 2015-2020, CIHI.

\section{Moving Forward}

This article illustrates the heavy toll that the COVID-19 pandemic has had on LTC residents during the first wave. Further investigation is required to better understand the unintended consequences of changes in care and limited contact with family and friends on LTC residents' physical and mental health. Although the situation in LTC has improved, CIHI will continue to work with partners, including the National Institute on Ageing and Statistics Canada, to help fill data gaps in the LTC sector and provide evidence to better inform actions that improve the quality of life for residents, staff and their families.

\section{References}

Canadian Institute for Health Information (CIHI). 2020, June. Pandemic Experience in the Long-Term Care Sector: How Does Canada Compare with Other Countries? Retrieved June 30, 2021. <https://www. cihi.ca/sites/default/files/document/covid-19-rapid-response-longterm-care-snapshot-en.pdf>.

Canadian Institute for Health Information (CIHI). 2021. The Impact of COVID-19 on Long-Term Care in Canada: Focus on the First 6 Months. Retrieved June 30, 2021. <https:/www.cihi.ca/sites/default/ files/document/impact-covid-19-long-term-care-canada-first-6months-report-en.pdf $>$.

Chu, C.H., J. Wang, C. Fukui, S. Staudacher, P.A. Wachholz and B. Wu. 2021. The Impact of COVID-19 on Social Isolation in Long-Term Care Homes: Perspectives of Policies and Strategies from Six Countries. Journal of Aging \& Social Policy. 33(4-5): 459-73. doi:10.1080/08959 420.2021 .1924346
Dudevich A. and J. Frood. 2021. Impact of the COVID-19 Pandemic on Health System Use in Canada. Healthcare Quarterly 24(2): 12-14. doi:10.12927/hcq.2021.26552.

Kemp, C.L. 2020. \#MoreThanAVisitor: Families as "Essential" Care Partners during COVID-19. The Gerontologist 61(2): 145-51. doi:10.1093/geront/gnaa161.

Stall, N.M., J. Johnstone, A.J. McGeer, M. Dhuper, J. Dunning and S.K. Sinha. 2020. Finding the Right Balance: An Evidence-Informed Guidance Document to Support the Re-Opening of Canadian Nursing Homes to Family Caregivers and Visitors during the Coronavirus Disease 2019 Pandemic. Journal of the American Medical Directors Association 21(10): 1365-70.e7. doi:10.1016/j.jamda.2020.07.038.

\section{About the Authors}

Raquel Betini, $\mathrm{PhD}, \mathrm{PhD}$, is a senior analyst at $\mathrm{ClHI}$ in Toronto, ON. She can be reached by e-mail at rbetini@cihi.ca.

Sandra Milicic, $\mathrm{PhD}$, is a senior analyst at $\mathrm{ClHI}$ in Toronto, ON.

Christina Lawand, MA, is a manager with Health System Analytics at $\mathrm{ClHI}$ in Ottawa, ON. 